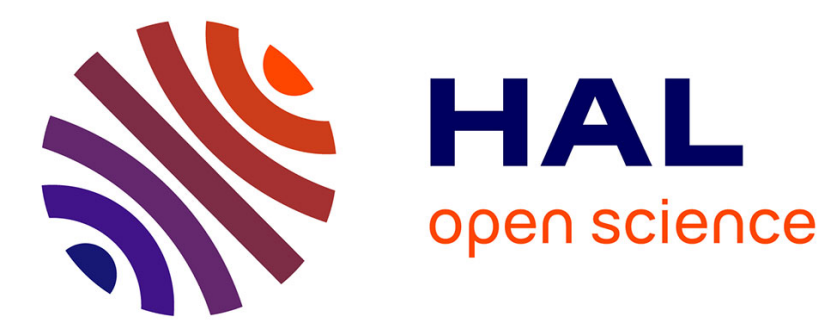

\title{
Stress fields in granular solids: Effect of composition
}

Vincent Topin, Farhang Radjai, Jean-Yves Delenne

\section{To cite this version:}

Vincent Topin, Farhang Radjai, Jean-Yves Delenne. Stress fields in granular solids: Effect of composition. Powder Technology, 2011, 208 (2), pp.568-573. 10.1016/j.powtec.2010.08.060 . hal-00689851

\section{HAL Id: hal-00689851 https://hal.science/hal-00689851}

Submitted on 27 Apr 2012

HAL is a multi-disciplinary open access archive for the deposit and dissemination of scientific research documents, whether they are published or not. The documents may come from teaching and research institutions in France or abroad, or from public or private research centers.

$$
\text { Copyright }
$$

L'archive ouverte pluridisciplinaire HAL, est destinée au dépôt et à la diffusion de documents scientifiques de niveau recherche, publiés ou non, émanant des établissements d'enseignement et de recherche français ou étrangers, des laboratoires publics ou privés. 


\title{
Stress fields in granular solids: effect of composition
}

\author{
Vincent Topin, Farhang Radjai, and Jean-Yves Delenne \\ LMGC UMR 5508 CNRS-Université Montpellier 2 / MIST, IRSN DPAM-CNRS, case \\ 048, Pl. E. Bataillon, 34095 Montpellier cedex 5, France
}

\begin{abstract}
We use the lattice element method to investigate stress fields at the subparticle scale in granular solids composed of particles embedded in a cementing matrix. The stress distributions are found to be similar in $2 \mathrm{D}$ and $3 \mathrm{D}$ samples subjected to vertical loading with free lateral boundaries. We find that the number of strong forces falls off exponentially at high particle volume fractions where a percolating network of jammed particles occurs. The influence of the matrix volume fraction and particle/matrix stiffness ratio with respect to stress distribution is analyzed in $2 \mathrm{D}$ and $3 \mathrm{D}$. We show that both decreasing the matrix volume fraction and increasing the stiffness ratio lead to increasingly broader distributions within a limit beyond which the distribution is independent of one or both of these parameters.
\end{abstract}

Keywords: granular solids, stress field, lattice element method,texture.

\section{Introduction}

Sedimentary rocks, biomaterials such as wheat endosperm, and geomaterials like mortars and concrete belong to the broad class of "cemented" granular materials [1-3]. They have in common a texture composed of particles embedded in a solid matrix of variable volume and cohesion with the 
particles. Their heterogeneity can be described either in terms of the composition (phase volume fractions, contacts and bonds between the particles, ...) or in terms of transport properties such as force transmission which basically reflect the details of the composition.

A considerable amount of experimental and numerical work has been devoted to force transmission in model granular media such as glass bead packs [4-9]. The force distributions are broad and highly heterogeneous. A pending issue is whether the presence of a particulate backbone is sufficient to produce similar heterogeneous force distributions in cemented granular media. A related issue is how a pore-filling solid matrix affects stress transmission, and in which respects it depends on the matrix volume fraction. It is not neither straightforward to generalize the force distributions under compressive loading to the case of tensile loading for a cohesive particle-matrix interface. In a similar vein, the role of particle stiffness and extended contact zones between the particles is important for the characterization of force transmission in cemented granular materials.

The investigation of this issue requires an approach in which the stresses can be resolved at sub-particle and sub-matrix scales. The lattice element method (LEM) has proved to be a reliable approach in this respect. It is based on a lattice-type discretization of the particles, matrix and their interface [1-3]. The elastic deformations of the particles are taken into account not only at their contacts with other particles or with the matrix, as in the discrete element method (DEM), but also in their bulk. The matrix is introduced with variable volume in the pores between the particles with its elastic properties and adhesion with the particles. 
In this paper, we use LEM to investigate the role of various parameters pertaining to the composition on stress transmission in $2 \mathrm{D}$ and $3 \mathrm{D}$ granular samples. In particular, we consider the effect of matrix volume fraction, particle stiffness and particle volume fraction. We also compare the force distributions in a packing simulated alternatively by LEM and DEM in the limit of low matrix volume fractions. Our findings indicate that the probability density of stresses carries a signature of the granular backbone that we will analyze in detail as a function of the matrix volume fraction and particle/matrix stiffness ratio.

\section{Numerical method and sample preparation}

The LEM is based on a discretization of the phases on a regular or irregular lattice. Hence, the space is represented by a grid of points (nodes) interconnected by one-dimensional elements (bonds). Each bond can transfer normal force, shear force and bending moment up to a threshold in force or energy, representing the cohesion of the phase or its interface with another

phase. Each phase (particle, matrix) and its boundaries are materialized by the bonds sharing the same properties. The samples are deformed by imposing displacements or forces to the nodes belonging to the contour. The total elastic energy of the system is a convex function of node displacements and thus finding the unique equilibrium configuration of the nodes amounts to a minimization problem. Performing this minimization for stepwise loading corresponds to subjecting the system to a quasistatic deformation process. The details of this method can be found in Ref. [1].

The samples are constructed either by geometric methods or by isotropic 
compaction of disk-like particles by DEM simulations by setting the friction coefficient between the particles to zero in order to get a dense packing. The samples are then discretized on a lattice. The matrix is introduced in the form of bridges of variable thickness, depending on the overall matrix volume fraction and the particle sizes, between neighboring particles throughout the system. As the matrix volume fraction is increased, the thickness of the bridges increases and eventually they merge to fill the interstitial space.

The elastic properties of each phase are controlled by the linear elastic properties of the bonds. The main elastic parameters that will be considered in this paper are the Hooke constants $k^{p}$ and $k^{m}$ of the bonds belonging to the particles and matrix, respectively. The initial state is the reference (unstressed) configuration. When the sample is loaded, bond forces develop inside the sample. A stress tensor $\sigma^{a}$ can be attributed to each node $a$ of the lattice network: $\sigma_{i j}^{a}=\frac{1}{V^{a}} \sum_{b} r_{i}^{a b} f_{j}^{a b}$ where the summation runs over all neighboring nodes $j, r_{i}^{a b}$ is the $i$ component of the vector joining the node $a$ to the midpoint of the bond $a b$ and $f_{j}^{a b}$ is the $j$ component of the bond force $[1,10]$.

The resolution of the stresses depends on the particle size compared to the lattice element lengths. The discretization should be sufficiently fine for the particle contours to be correctly represented. The macroscopic elastic moduli might crucially depend on the discretization as more generally in porous materials $[11,12]$. In practice, however, the resolution is set as a result of compromise between the necessary number of particles for statistical representativity and total number of nodes accessible to computer simulation. In the simulations reported in this paper, we generally favored high resolution 
both in 2D and 3D simulations such that the results for stress transmission reliably reflect the configuration of the particle phase.

In the following, we mainly consider node stresses in rectangular and cubic samples subjected to vertical loading with free lateral boundaries. At low matrix volume fractions, for comparison with DEM we will also evaluate the contact forces between particles from bond forces. During loading, the bond forces increase with the applied vertical stress at the boundary. Hence, the mean bond force increases linearly with the external load whereas the probability density functions (pdf's) of bond forces and stresses do not evolve as long as no bond breaks. In this paper, we focus only on force distributions in the undamaged samples, i.e. in the purely elastic domain. The damage and fracture properties have been extensively studied elsewhere [1].

\section{Sub-particle stresses and contact forces}

In order to obtain fine statistics of node stresses and contact forces between particles, we simulated a large sample of about 5000 particles with a particle volume fraction of $\rho^{p} \simeq 0.8$. This corresponds to a packing with a dense contact network of coordination number $z=4$. The particle diameter $d$ varies between $d_{\min }$ and $d_{\max }=3 d_{\min }$ with a uniform distribution by volume fractions $\left(P(d) \propto d^{2}\right)$.

[Figure 1 about here.]

We would like to compare the contact forces in this system, simulated by the LEM, with those in a similar system simulated by the DEM. This can be done only in the limit of a small matrix volume fraction where the matrix 
is found in the form of small solid bridges between the particles such that its effect can be represented by a cohesion law. We used a matrix volume fraction of $\rho^{m} \simeq 0.01$. The DEM code is based on the standard molecular dynamics method with cohesive bonding between the particles; see Ref. [13]. The sample is subjected to vertical compression.

Figure 1 shows the vertical stress field $\sigma_{y y}$. The node stresses are represented by proportional color levels over the elementary hexagonal cells centered on each node. We observe chains of highly stressed particles and higher concentration at the contact zones between the particles. In order to compare the LEM simulated packing with DEM simulations of the same packing, for which only contact forces are accessible, we compute the contact forces $\vec{f}$ by summing up the bond forces $\overrightarrow{f^{a b}}$ for all bonds $a b$ crossing the contact plane $S: \vec{f}=\sum_{a b \in S} \overrightarrow{f^{a} b}$.

Figure 2 shows the map of normal forces between particles for the LEM and DEM packings. We observe very similar force chains despite the fact that radically different methods were used to simulate them. The Pearson product-moment correlation coefficient between the two force networks is $r=0.92$, which indicates high similarity. The probability density functions (pdf's) of normal and tangential forces from LEM and DEM simulations are shown in Fig. 3. We observe that the two pdf's coincide over nearly the whole range of forces.

This agreement between the two methods is all the more interesting that in DEM the particles are assumed to be rigid and the stresses inside the particles are not involved in the calculation of contact forces. The pdf has the well-known features of force distributions in dry granular media The forces 
below the mean have a nearly uniform distribution whereas larger forces represent a nearly exponential decay: $P_{f}\left(f_{n}\right) \propto e^{-\beta f_{n} /\left\langle f_{n}\right\rangle}$. This excellent agreement between the force pdf's with $\beta \simeq 1.35$ may be considered as a validation of DEM results for the force networks in the sense that the contact forces in LEM simulations are calculated from a finer scale $[5,8,9]$.

[Figure 2 about here.]

[Figure 3 about here.]

[Figure 4 about here.]

Having access to the node stresses, it is interesting to evaluate their pdf's and to see if they carry a signature of the composition. One example of the pdf of vertical stresses $\sigma_{y y}$ is displayed in Fig. 4(a) for a packing under vertical compression. Since the sample is under axial compression, only $4 \%$ of vertical stresses are tensile and are thus not shown in Fig. 4(a). The strong stresses fall off exponentially as contact forces (see Fig. 3), $P_{\sigma}\left(\sigma_{y y}\right) \propto e^{-\beta \sigma_{y y} /\left\langle\sigma_{y y}\right\rangle}$ with $\beta \simeq 0.95$, and they mostly concentrate at the contact zones. The weak stresses have a nonzero pdf, much the same as weak contact forces, reflecting the arching effect. Since the contact force distributions reflect the granular disorder, i.e. the structure of the network of contiguous particles, the observed similarity between the distributions of stresses and forces means that the sub-particle stresses are strongly affected by the granular disorder.

[Figure 5 about here.]

[Figure 6 about here.] 


\section{Effect of composition in $2 \mathrm{D}$}

\subsection{Matrix volume fraction}

It is expected that at higher matrix contents the stress is more homogeneously redistributed inside the packing due to load transfer between the particles and the matrix. Fig. 5 shows $P_{\sigma}$ for three values of $\rho^{m}$ in tension and compression for $k^{p}=100 k^{m}$. Interestingly, the exponential tail persists both in tension and in compression, but for equal matrix volume fractions, the pdf of strong stresses is broader in compression than in tension. In other words, the stress redistribution is more homogeneous in tension than in compression.

It is also interesting to observe that the stress pdf is not affected by the matrix volume fraction in compression but it is increasingly broader in tension for decreasing matrix content so that the stresses are more and more concentrated in the bridges between the particles. In tension, the exponent $\beta$ varies from 1.10 to 2.55 as $\rho^{m}$ varies from 0.08 to 0.12 whereas in compression we have $\beta \simeq 0.95$ for all $\rho^{m}$. As $\rho^{m}$ increases, the gaussian peaked on the mean stress, corresponding mainly to the stresses in the bulk of the particles, becomes more and more pronounced.

\subsection{Particle/matrix stiffness ratio}

We now consider the influence of the particle/matrix stiffness ratio $k^{p} / k^{m}$ on stress distribution. Fig. 6 displays the vertical stress pdf's for three values of $k^{p} / k^{m}$ in tension and compression for $\rho^{m}=0.10$. It is remarkable that in tension the particle stiffness has little influence on the pdf whereas in compression the pdf becomes increasingly broader for an increasing particle 
stiffness. The respective effects of particle stiffness and matrix volume fraction can be understood by remarking that, due to the presence of a granular backbone, the stress chains are essentially guided by the cementing matrix in tension and by the particle phase in compression. Therefore, the stress transmission is not affected by the matrix volume fraction in compression and only slightly influenced by particle stiffness in tension.

[Figure 7 about here.]

[Figure 8 about here.]

\subsection{Particle volume fraction}

The jamming of particles is an important consequence of high particle volume fraction in cemented granular materials. Jamming has profound effects on the mechanical response in terms of stress concentration, micro-cracking, and sensitivity to loading parameters [14-16]. In particular, the arching effect leads to weakly stressed zones in the bulk reflected in the pdf of weak forces. The issue that we would like to address here is how sensitive the force pdf's are with respect to jamming.

If the particle volume fraction is sufficiently high, a dense skeleton of contiguous particles naturally occurs. But when the particle volume fraction is reduced, the contact network becomes more sparse, and at still lower particle volume fractions the contact network disappears. In this limit, all adjacent particles are interposed by matrix ligaments. This is what we observe in Fig. 8 where four small samples of particle volume fractions $\rho^{p}=0.65,0.7,0.75,0.8$ are shown together with the corresponding networks of contacting particles. Only in the two last samples with $\rho^{p} \geq 0.75$ a percolating network of contacts 
can be observed. Fig 7 shows the vertical stress pdf's in these samples under axial compression. In order to reduce the effect of matrix volume fraction on the results, we set its value to maximum (zero porosity). As expected, the pdf's are increasingly broader for an increasing particle volume fraction. This means that stresses are more and more localized in the contacts between particles. But their form is rather gaussian as long as no percolating contact network is present. A qualitative change occurs at $\rho^{p}=0.75$, and the pdf becomes exponential in the range of strong forces.

\section{Effect of composition in $3 \mathrm{D}$}

In this section, we briefly extend our studies to 3D cemented granular solids. We use a 3D version of LEM in which the particles, matrix and interfaces are materialized by 1D elements forming a 3D lattice. We generated a dense packing of 300 particles discretized over an irregular lattice containing about 500000 elements. The particle diameters $d$ vary between $d_{\min }$ and $d_{\max }=2 d_{\min }$ with a uniform distribution by volume fractions. The particle volume fraction is $\rho^{p} \simeq 0.63$. As in our 2D LEM simulations, the matrix is distributed in the form of bridges between neighboring particles. The sample is displayed in Fig. 9. This protocol allows us to vary the matrix volume fraction continuously from 0 to 0.37 . Here, we focus on the trends. The details of a full parametric study will be published elsewhere.

[Figure 9 about here.]

[Figure 10 about here.]

[Figure 11 about here.] 
[Figure 12 about here.]

Fig. 10 shows the vertical stresses on a cut plane for three values of $\rho^{m}$. We observe that the stresses are more and more localized in the matrix bridges when the matrix volume fraction decreases. Figure 12 shows the vertical stress pdf's for three values of $k^{p} / k^{m}$ and three values of $\rho^{m}$ under vertical compression with free lateral boundaries. Two limits are of particular interest: (1) The homogeneous limit characterized by $\rho^{m}=0.37$ and $k^{p}=k^{m}$, corresponding to a homogeneous material with no void and no particle; (2) The granular limit characterized by large $k^{p}$ and no matrix $\left(\rho^{m} \simeq 0\right)$ or sufficiently weak matrix volume fraction basically distributed in the form of small solid bonds between particles, corresponding to a granular material with stiff particles as generally assumed in DEM simulations. We see that, as expected, the stress distribution for the homogeneous limit is the less broad one with a nearly gaussian shape. The stress variability in this system reflects the metric disorder of the underlying lattice. The distribution for $\rho^{m}=0.1$ and $k^{p} / k^{m}=100$ corresponds to the granular limit. We observe the nearly exponential fall-off of strong stresses together with a peak on a value slightly below the mean stress as in $2 \mathrm{D}$ simulations and noncohesive granular materials simulated by DEM.

The distribution in the granular limit is practically the broadest one, and hence all distributions for all parameters lie between those for the granular and homogeneous limits. For $\rho^{m}=0.23$ and $k^{p} / k^{m}=1$ we have a porous material with no mechanical contrast between the matrix and particles. For $\rho^{m}=0.37$ and $k^{p} / k^{m}=50$ we have a granular phase embedded in a matrix with no voids. In both these cases, the stress distribution is broader than 
that in the homogeneous limit although the physical origins of this enhanced inhomogeneity are different. We remark that, for $\rho^{m}=0.1$, increasing $k^{p} / k^{m}$ from 50 to 100 has little influence on the stress distribution. In the same way, for $k^{p} / k^{m}=50$, increasing $\rho^{m}$ from 0.1 to 0.23 has practically no impact on the distribution.

Concerning the weak stresses, we observe that the stress pdf vanishes in the absence of particle-matrix contrast, i.e. when no granular disorder is present, as the stress tends to zero. A secondary peak is observed in the range of weak stresses in the presence of a granular disorder. Our data indicate that this peak reflects the weak stresses inside the matrix bridges, as observed in Fig. 11. The lower the peak, the more homogeneous is stress transmission through the matrix bridges.

\section{Conclusion}

The lattice element method provides a suitable framework for the investigation of stress fields in complex granular solids involving a solid matrix sticking to the particles. Using this method, and by coarse-graining the sub-particle stresses, we arrive at the same contact force distributions as those commonly obtained by DEM simulations and experiments. Our data are consistent with the fact that the decreasing exponential distribution of strong forces is a signature of granular disorder, i.e. the disorder induced by a contiguous network of stiff particles. This signature disappears in the homogeneous limit where there is no stiffness contrast between the particle and matrix phases and the porosity vanishes or when the particles are interposed everywhere by the cementing matrix. The 3D simulations evidence the two 
limits of homogeneous and granular texture and the effect of the composition on the distributions as the texture deviates from these two limits.

[1] V. Topin, J.-Y. Delenne, F. Radjaï, L. Brendel, F. Mabille, Strength and fracture of cemented granular matter, The European Physical Journal E 23 (2007) 413-429.

[2] V. Topin, F. Radjai, J.-Y. Delenne, A. Sadoudi, F. Mabille, Wheat endosperm as a cohesive granular material, Journal of Cereal Science 47 (2) (2008) 347-356.

[3] E. Schlangen, E. J. Garboczi, Fracture simulations of concrete using lattice models: Computational aspects, Engineering Fracture Mechanics 57 (2-3) (1997) 319-332.

[4] C. h. Liu, S. R. Nagel, D. A. Schecter, S. N. Coppersmith, S. Majumdar, O. Narayan, T. A. Witten, Force fluctuations in bead packs, Science 269 (5223) (1995) 513-515.

[5] F. Radjaï, M. Jean, J. J. Moreau, S. Roux, Force distribution in dense two-dimensional granular systems, Phys. Rev. Lett. 77 (2) (1996) 274.

[6] H. M. Jaeger, S. R. Nagel, R. P. Behringer, Granular solids, liquids, and gases, Rev. Mod. Phys. 68 (4) (1996) 1259-.

[7] F. Radjaï, D. E. Wolf, M. Jean, J. J. Moreau, Bimodal character of stress transmission in granular packings, Phys. Rev. Lett. 80 (1) (1998) $61-64$. 
[8] D. M. Mueth, H. M. Jaeger, S. R. Nagel, Force distribution in a granular medium, Phys. Rev. E 57 (1998) 3164.

[9] T. S. Majmudar, R. P. Behringer, Contact force measurements and stress-induced anisotropy in granular materials, Nature 435 (1079) (2005) 1079-1082.

[10] J. J. Moreau, Numerical investigation of shear zones in granular materials, in: D. E. Wolf, P. Grassberger (Eds.), Friction, Arching, Contact Dynamics, World Scientific, Singapore, 1997, pp. 233-247.

[11] A. Roberts, E. J. Garboczi, Elastic properties of model random threedimensional open-cell solids, J. Mech. Phys. Solids 50 (2002) 33-55.

[12] E. J. Garboczi, A. R. Day, An algorithm for computing the effective linear elastic properties of heterogeneous materials: three- dimensional results for composites with equal phase poisson ratios, J. Mech. Phys. Solids 43 (1995) 1349-1362.

[13] H. Peron, J. Delenne, L. Laloui, M. El Youssoufi, Discrete element modelling of drying shrinkage and cracking of soils, Computers and Geotechnics 36 (1-2) (2008) 61-69.

[14] H. J. Herrmann, S. Roux (Eds.), Statistical Models for Fracture in Disordered Media, North Holland, Amsterdam, 1990.

[15] D. Elata, J. Dvorkin, Pressure sensitivity of cemented granular materials, Mechanics of Materials 23 (2) (1996) 147-154. 
[16] A. J. Liu, S. R. Nagel (Eds.), Jamming And Rheology, Taylor and Francis, New York, 2001. 


\section{List of Figures}

1 Vertical stress field $\sigma_{y y}$ represented in color level. . . . . . . . 17

2 A map of normal forces in a portion of a sample under vertical compression simulated by DEM (a) and LEM (b). Line thickness is proportional to the normal force. Very weak and tangential forces are not shown. . . . . . . . . . . . . . 18

3 Probability density function of normal forces (a) and tangential forces (b) in a sample axially compressed by LEM and DEM simulations. The forces are normalized by the mean normal force. . . . . . . . . . . . . . . . . . . . . 19

4 Probability density function of vertical stresses normalized by the average stress in compression. . . . . . . . . . . . . 20

5 Probability density functions of normalized vertical stresses for three values of the matrix volume fraction (a) in tension and (b) in compression. . . . . . . . . . . . . . 21

6 Probability density functions of normalized vertical stresses for three values of the relative stiffness $k^{p} / k^{m}$ (a) in tension and (b) in compression. . . . . . . . . . . . . . . 22

$7 \quad$ Probability density functions of normalized vertical stresses for five values of the particle volume fraction $\rho^{p}$ in compression. 23

8 Four samples with four different particle volume fractions: 0.65 (a), 0.7 (b), 0.75 (c) and 0.8 (d). The grey particles are in contact with at least another particle. . . . . . . . . . . 24

9 (Color online) Representation of a cemented granular sample composed of particles (in red), interfaces (in green) and matrix (in bleu) discretized on a 3D irregular lattice. . . . . . . . . 25

10 (Color on line) Vertical stresses field $\sigma_{y y}$ represented in 3D on a cut plane in color level for (a) $r h o^{m}=0.37$, (b) $r h o^{m}=0.23$,

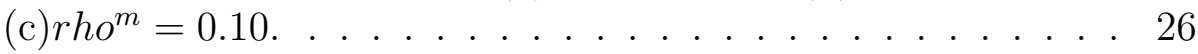

11 Probability density functions of normalized vertical stresses for $\rho^{m}=0.1$ and $k^{p} / k^{m}=50$ in the particle and matrix phases in comparison with that in the whole sample. . . . . . . 27

12 Probability density functions of normalized vertical stresses for different values of stiffness ratio $k^{p} / k^{m}$ and values of the matrix volume fraction $\rho^{m}$ in compression. . . . . . . . 28 


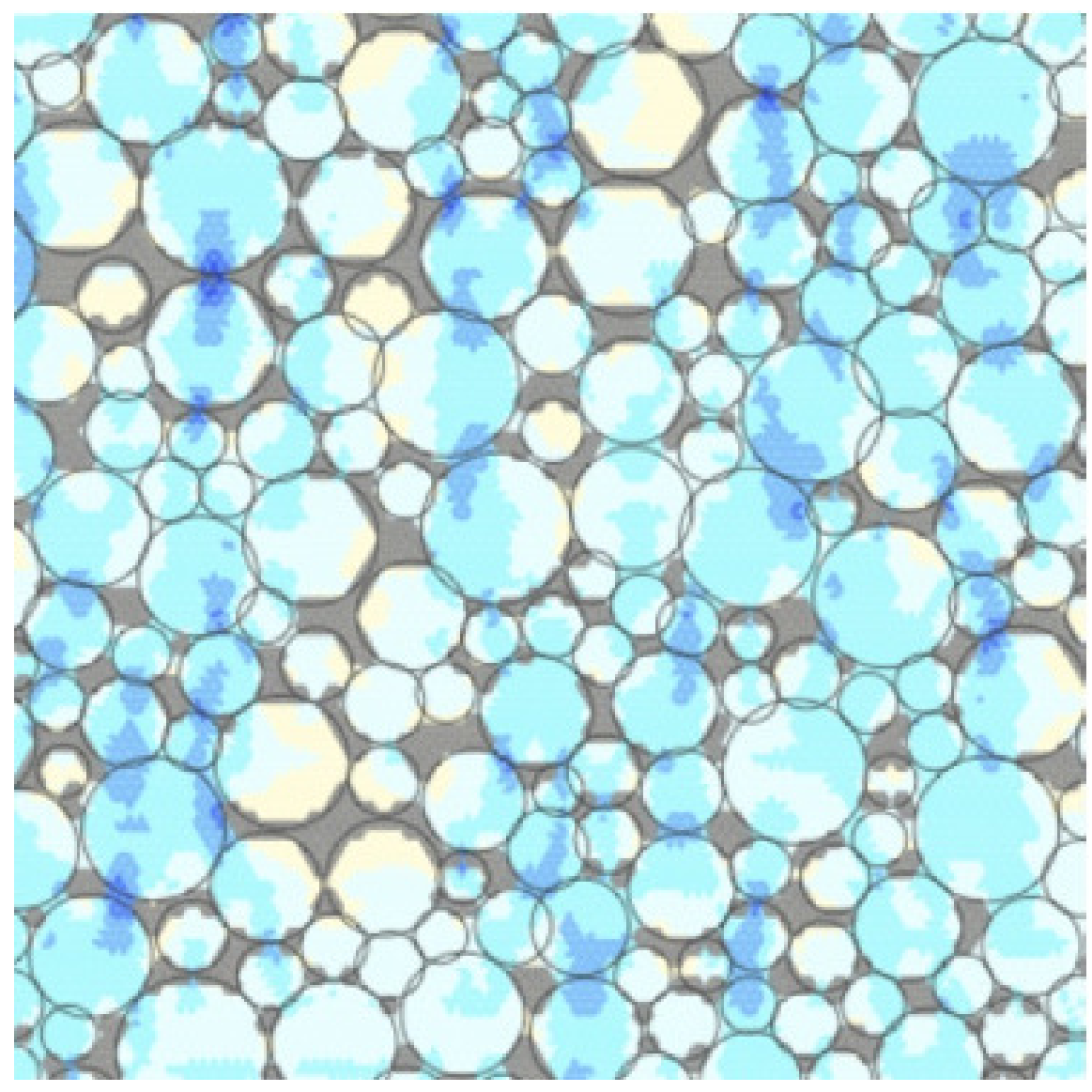

Figure 1: Vertical stress field $\sigma_{y y}$ represented in color level. 


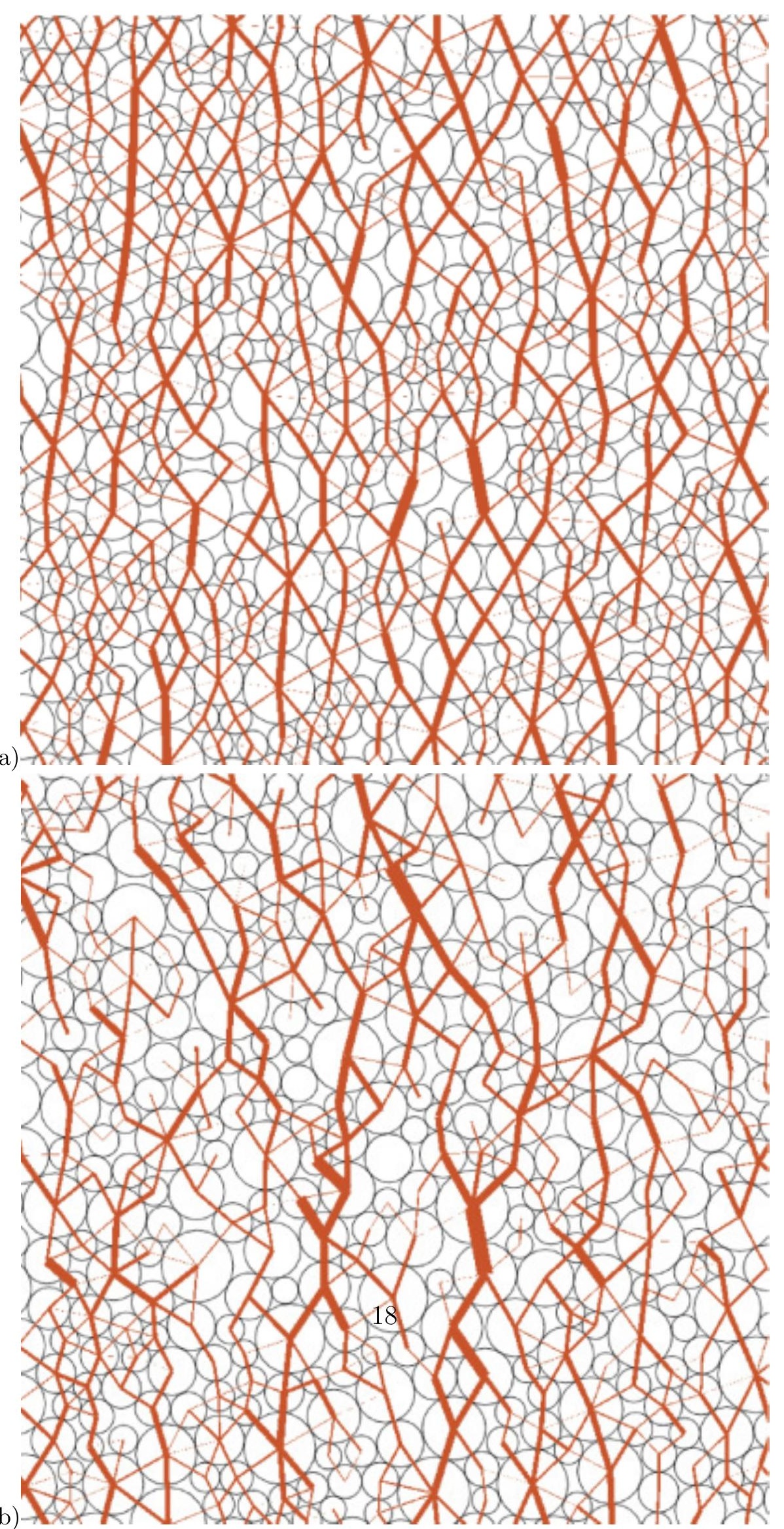



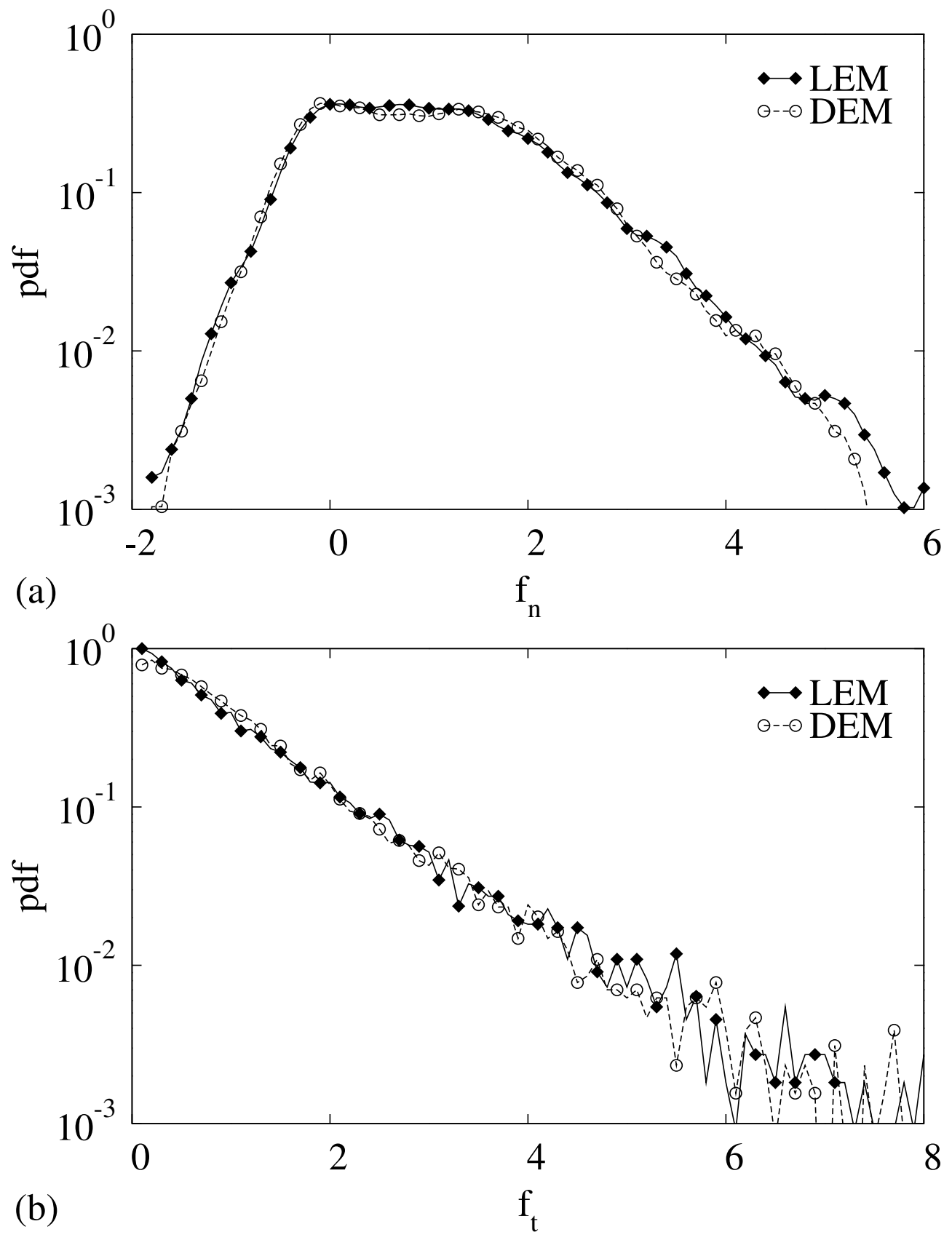

Figure 3: Probability density function of normal forces (a) and tangential forces (b) in a sample axially compressed by LEM and DEM simulations. The forces are normalized by the mean normal force. 


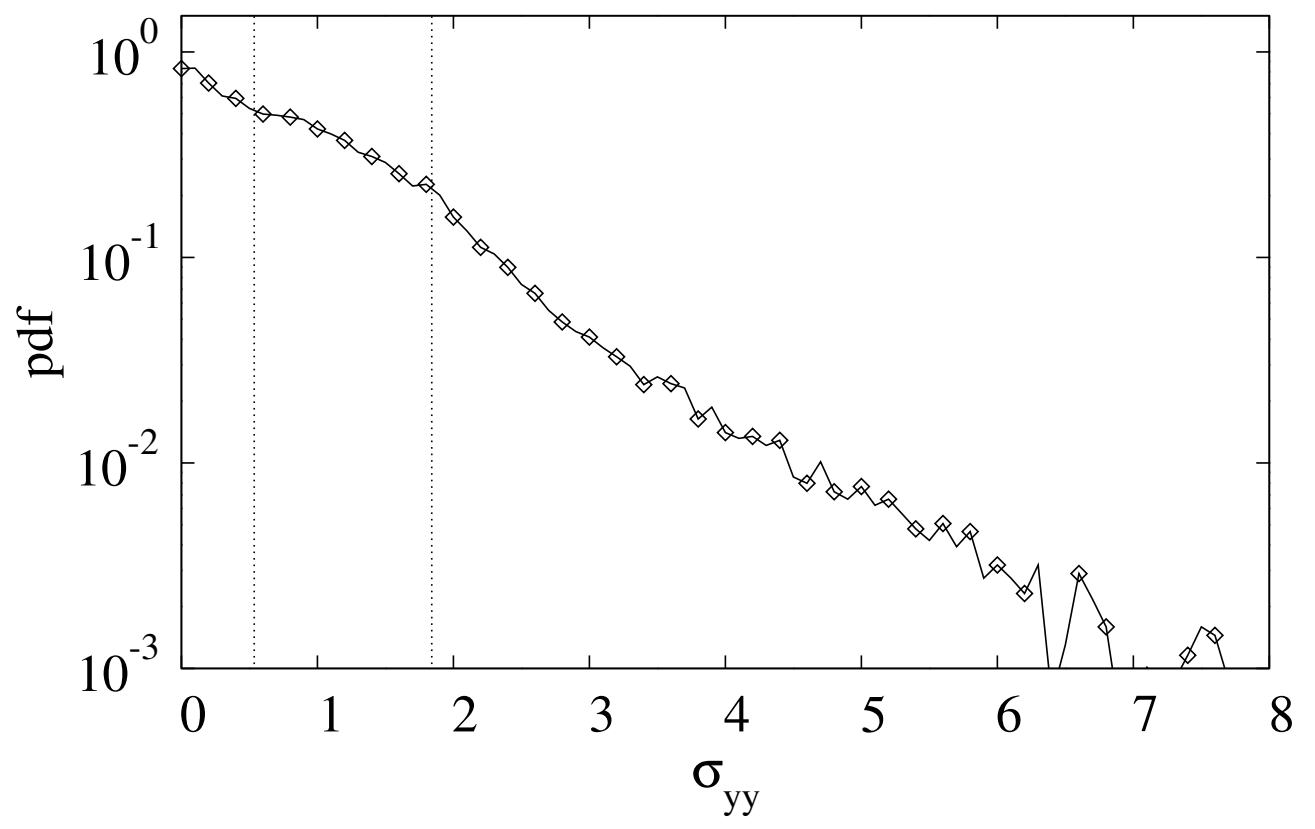

Figure 4: Probability density function of vertical stresses normalized by the average stress in compression. 

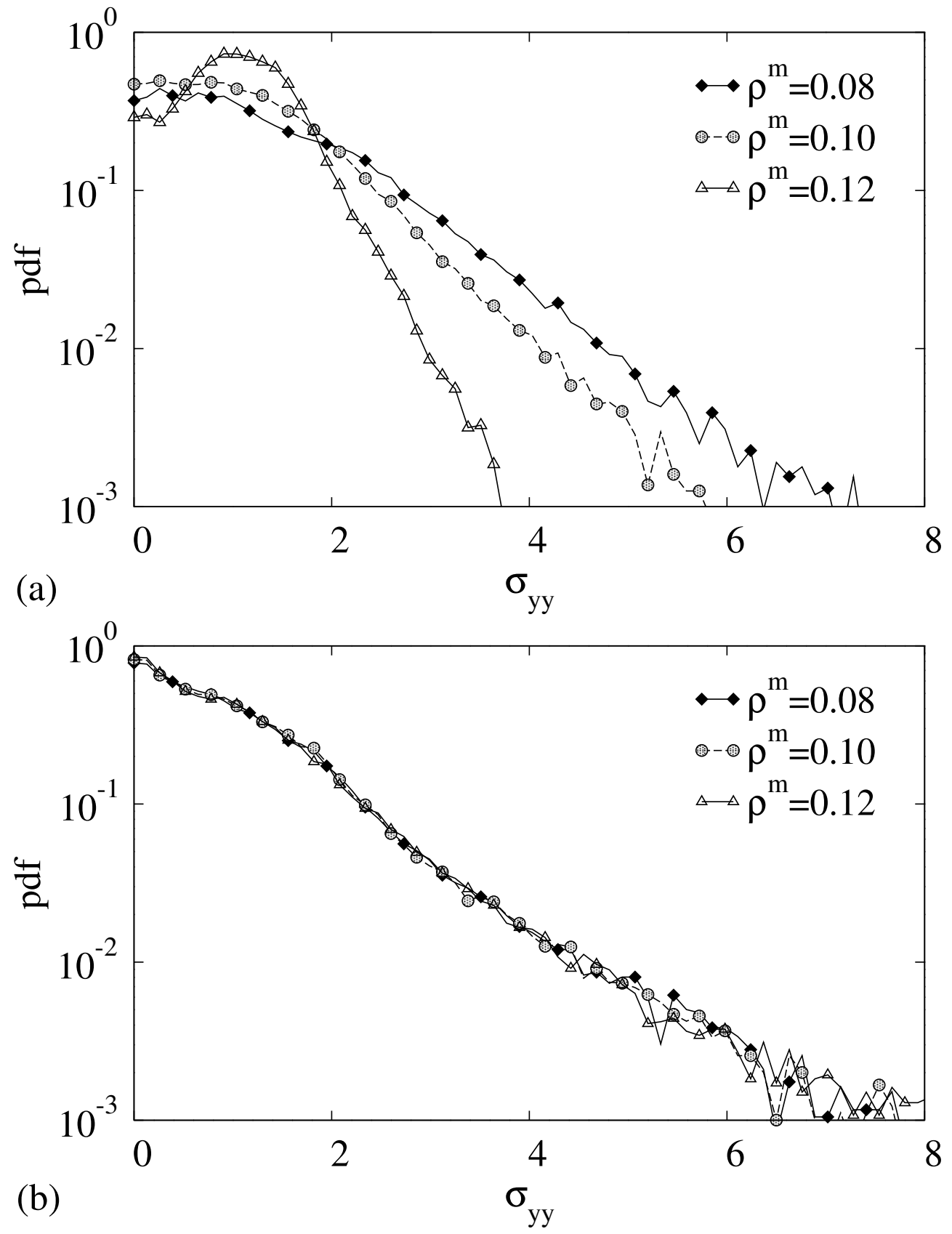

Figure 5: Probability density functions of normalized vertical stresses for three values of the matrix volume fraction (a) in tension and (b) in compression. 

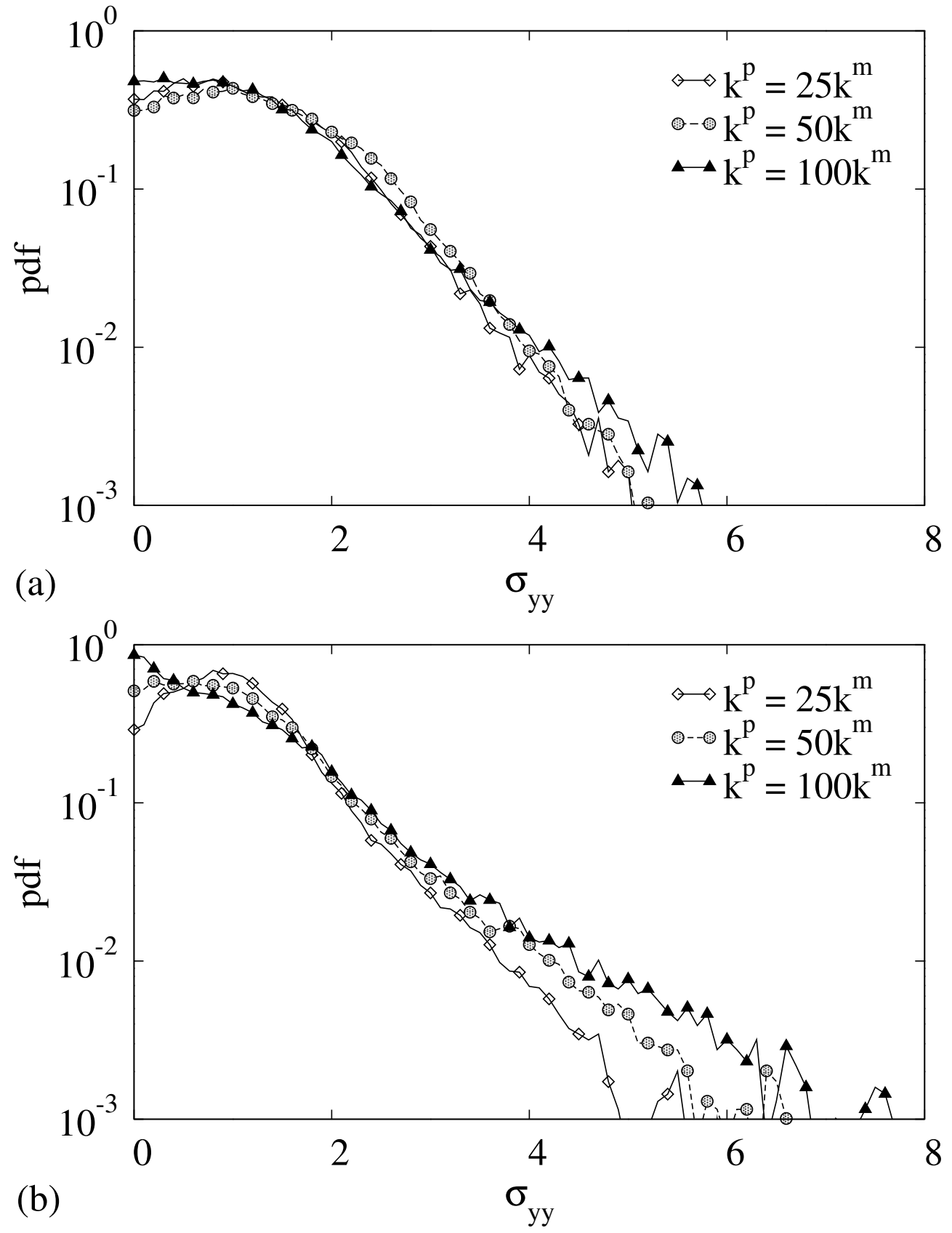

Figure 6: Probability density functions of normalized vertical stresses for three values of the relative stiffness $k^{p} / k^{m}$ (a) in tension and (b) in compression. 


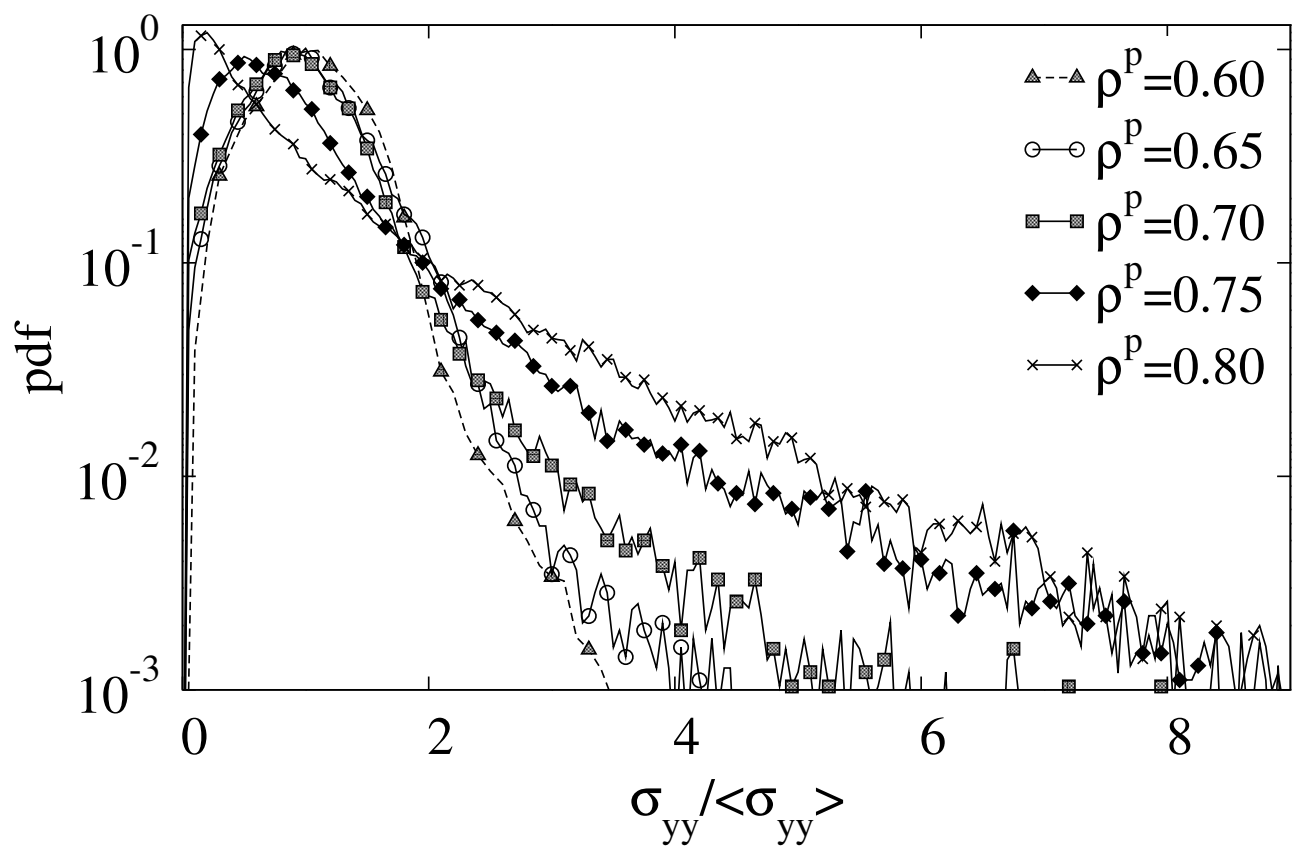

Figure 7: Probability density functions of normalized vertical stresses for five values of the particle volume fraction $\rho^{p}$ in compression. 

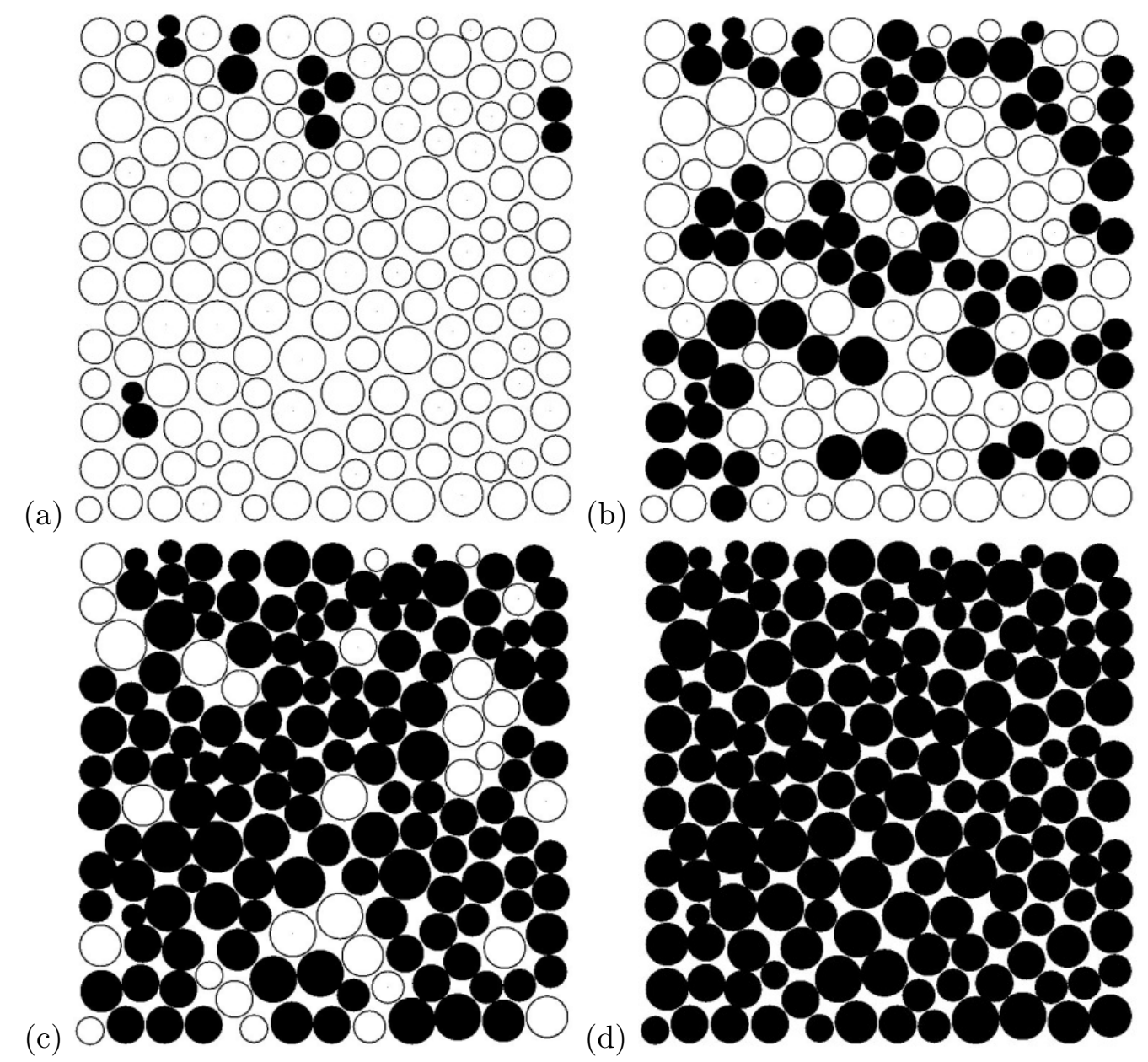

(b)

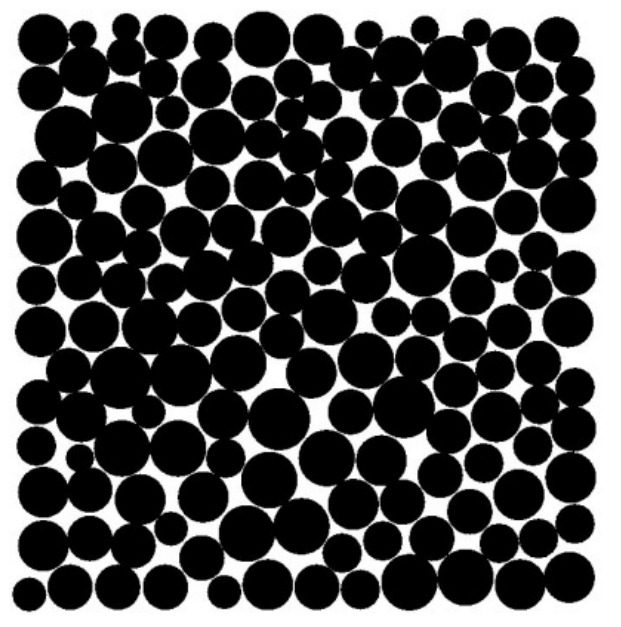

Figure 8: Four samples with four different particle volume fractions: 0.65 (a), 0.7 (b), 0.75 (c) and $0.8(\mathrm{~d})$. The grey particles are in contact with at least another particle. 


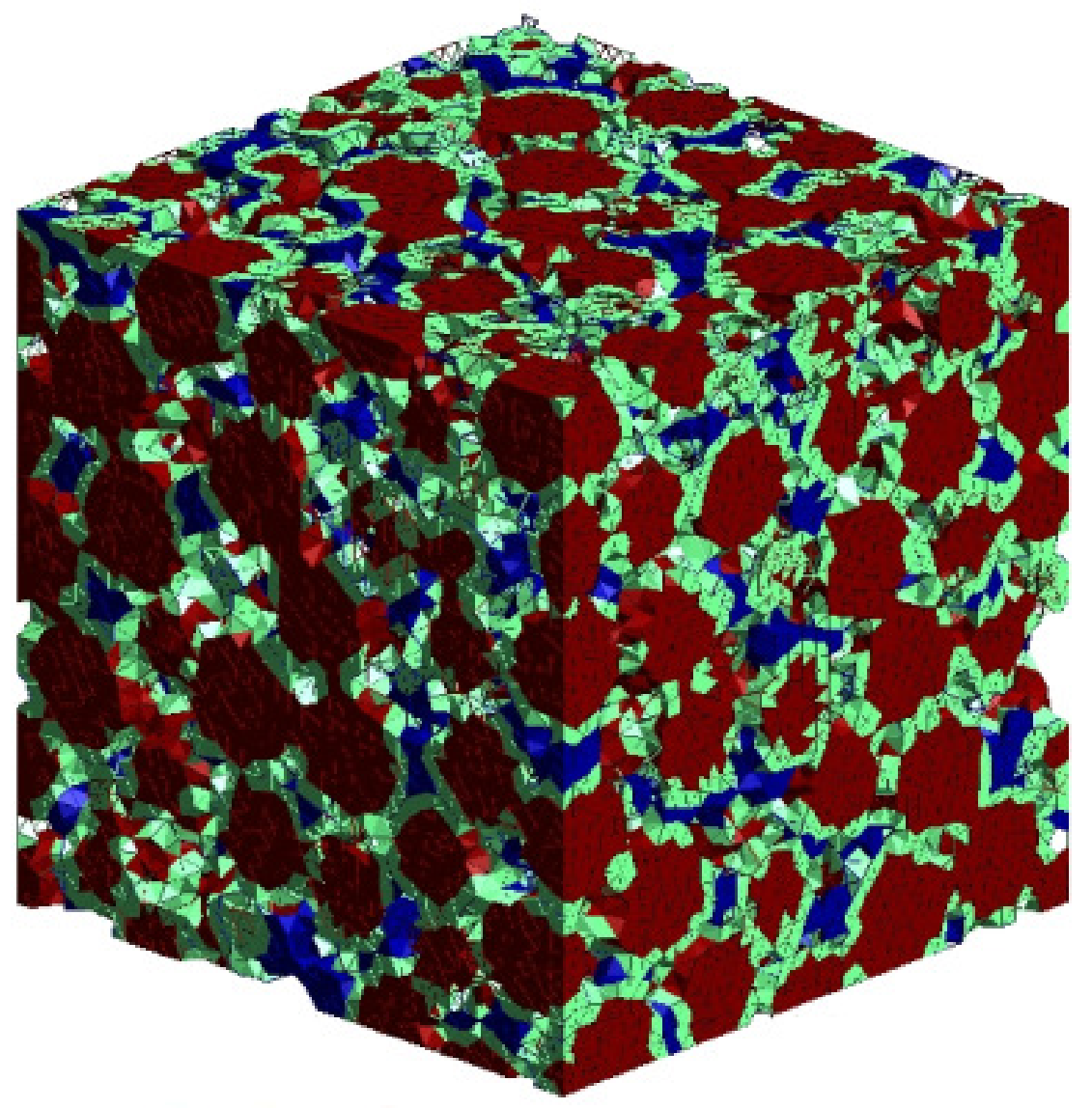

Figure 9: (Color online) Representation of a cemented granular sample composed of particles (in red), interfaces (in green) and matrix (in bleu) discretized on a 3D irregular lattice. 


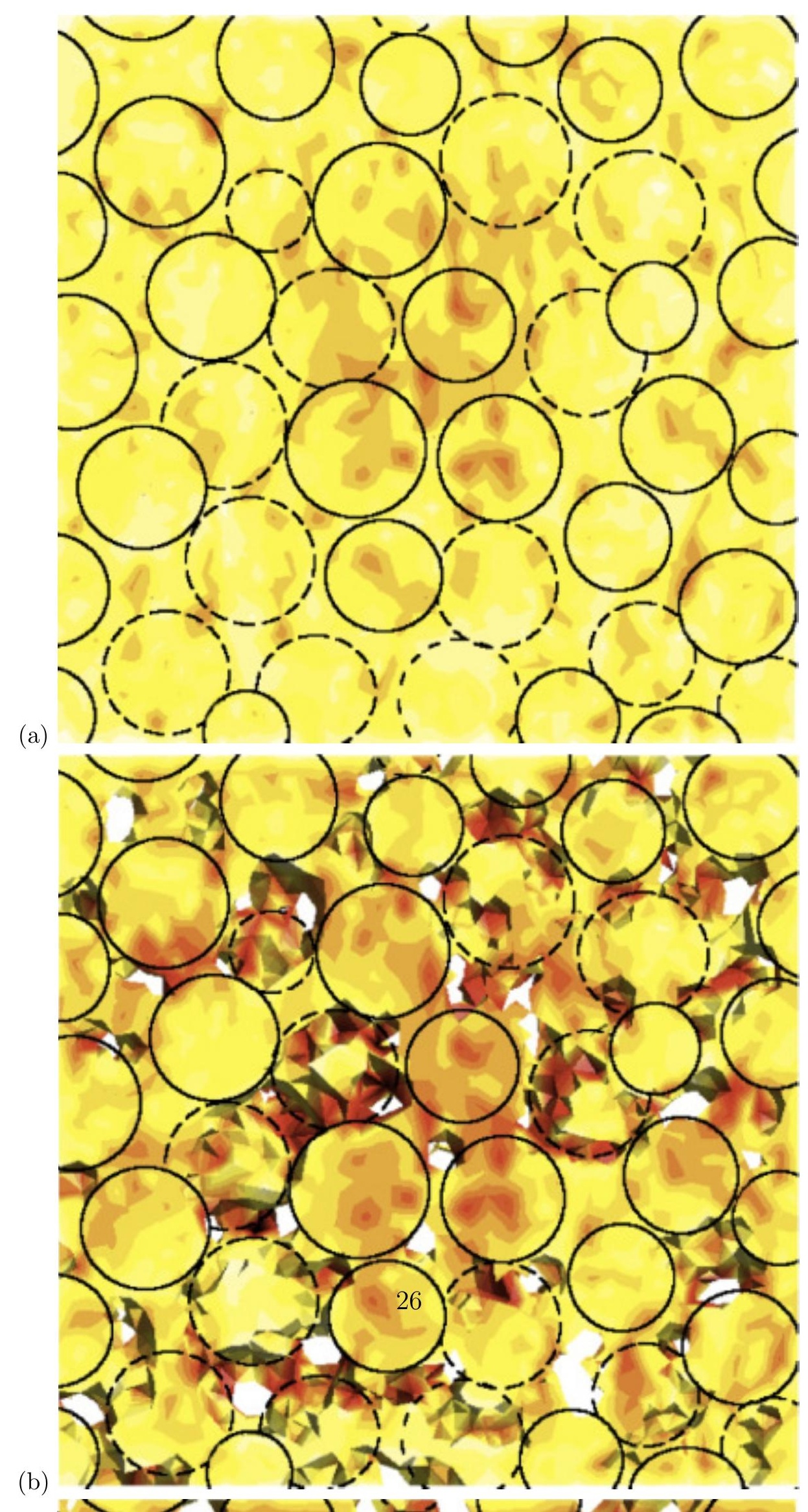




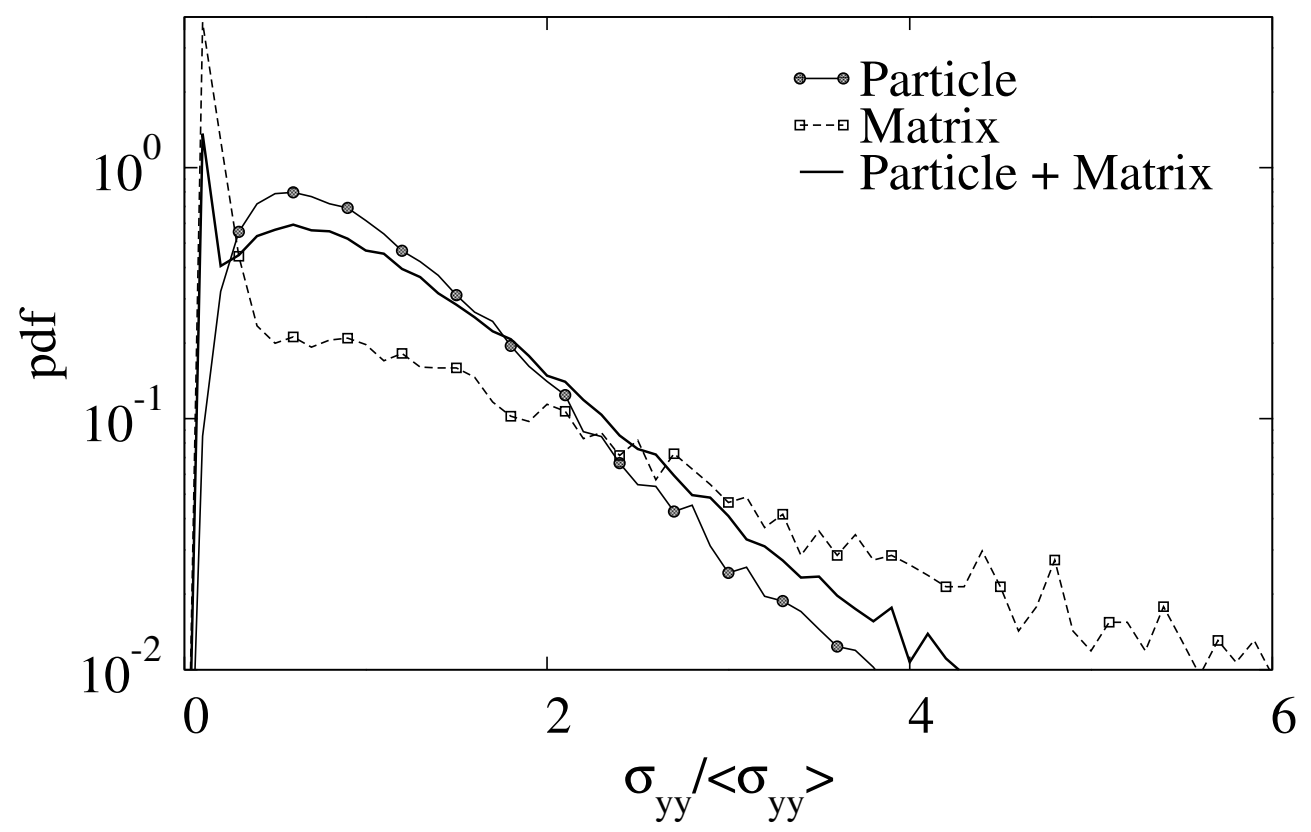

Figure 11: Probability density functions of normalized vertical stresses for $\rho^{m}=0.1$ and $k^{p} / k^{m}=50$ in the particle and matrix phases in comparison with that in the whole sample. 


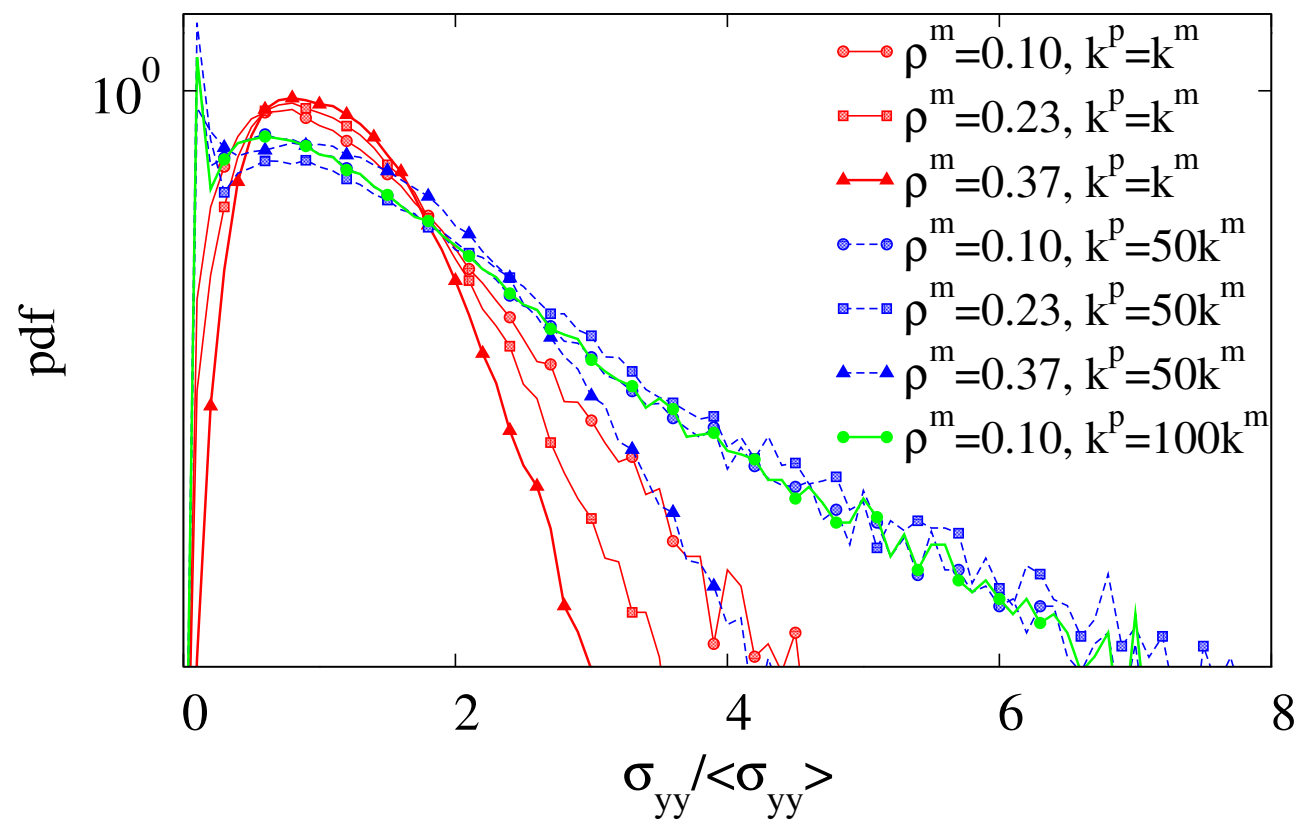

Figure 12: Probability density functions of normalized vertical stresses for different values of stiffness ratio $k^{p} / k^{m}$ and values of the matrix volume fraction $\rho^{m}$ in compression. 